\title{
Direct Position Determination of Coherently Distributed Noncircular Sources
}

\author{
Zhiyu Lu $\mathbb{D}^{D}$, Jianhui Wang $\mathbb{D}^{D}$, Bin Ba $\mathbb{D}^{D}$, and Daming Wang \\ China National Digital Switching System Engineering \& Technological R\&D Center, Zhengzhou 450000, China \\ Correspondence should be addressed to Jianhui Wang; 18537123322@163.com
}

Received 28 December 2017; Revised 18 April 2018; Accepted 3 May 2018; Published 4 June 2018

Academic Editor: Cheeyen Leow

Copyright (C) 2018 Zhiyu Lu et al. This is an open access article distributed under the Creative Commons Attribution License, which permits unrestricted use, distribution, and reproduction in any medium, provided the original work is properly cited.

The existing localization algorithms for coherently distributed (CD) sources fail to achieve the best localization performance because of position information loss and error accumulation. To solve this problem, this study proposed a novel direct position determination (DPD) algorithm that profits from the characteristics of noncircular signals. Based on the parameterization assumption of CD sources, the DPD localization model is initially constructed and an extended subspace data fusion-based DPD algorithm is subsequently proposed by decomposing the extended covariance matrices, which are constructed by combining the characteristics of noncircular sources. The algorithm achieves a low complexity and a high efficiency by avoiding the calculation of the intermediate variables. Specifically, the closed-form expression of the Cramer-Rao lower bound for CD noncircular sources is also derived. Simulation results show that, compared with existing DPD algorithms, the proposed algorithm improves the multitarget localization capability and achieves high-accuracy results.

\section{Introduction}

Wireless target location technology is of considerable value in emergency rescue, safety management, and navigation planning. Numerous studies on this subject have indicated that this field has attracted the attention of scholars in the academe and industry [1-3]. In most existing localization algorithms, the target is modeled as a point source, which has the advantages of simple calculation and high precision [4-7]. However, in location applications, such as cellular mobile communication, low elevation radar, and underwater positioning, the target is usually crowded with surrounding scatterers that reflect the signal toward the receiving array. In such case, the target is not perceived as a point but rather as a spatial coherently distributed (CD) source, and the received signals are composed of multiple paths with angular expansion [8-10]. If the point source model is still used for this condition, then the algorithm will rarely provide a high fidelity location result. The point source model is ideal, whereas the CD source model is closer to the actual scene. Therefore, analyzing the localization method for CD sources is more practically useful than that for point sources.
Obtaining the CD source position in a complex environment is difficult. Thus, the parameterization assumption combined with spatial distribution characteristics is often used to facilitate theoretical analysis. Jantti first proposed the concept of spatially distributed source and used the angle density function to reflect the angle expansion characteristics of received signals [11, 12]. Based on this concept, the Vec-MUSIC [13], DSPE [14], and ESPRIT [15] algorithms have been designed to enable parameter estimation of $\mathrm{CD}$ sources. As the special characteristics of noncircular signals, such as the common communication signals like binary phase shift keying (BPSK), amplitude modulation (AM), and preprocessed minimum shift keying (MASK), can improve estimation performance, the study of noncircular signals is of considerable practical value. Thus, Yang proposed a method for CD noncircular signals based on sparse representation, which exhibits a good performance under the condition of small snapshots with low SNR [16]. Wan put forward a DSPE algorithm based on the combination of circular and noncircular signals in a large MIMO system, which improves the parameter estimation precision [17]. To reduce computation cost, Yang combined the characteristics of noncircular signals 
with the ESPRIT algorithm to avoid complex parameter search and obtain high-accuracy results [18]. Compared with the algorithms of circular signals, the algorithms based on the characteristics of noncircular signals enhance the parameter estimation accuracy of CD sources significantly.

After achieving parameter estimation of $\mathrm{CD}$ sources, target localization based on certain methods, such as Chan algorithm, Taylor series expansion, and Kalman filter, should be accomplished. The above-mentioned conventional localization methods employ two-step processing, whereby the measurement parameters (e.g., AOA and TOA) are initially extracted, and the source positions are subsequently estimated [19]. The two-step method has several shortcomings. In the first step, the measurement parameters are acquired separately and independently from each receiver, disregarding the intrinsic constraint that the measurements are consistent with the same target position, thereby leading to loss of location information. In the second step, parameter estimation accuracy is greatly influenced by complex environment, and it is not easy to get high-accuracy estimation results, which inevitably involves processing errors that affect positioning accuracy. Therefore, the two-step method is suboptimal, failing to achieve the best performance [19]. Compared with the two-step localization algorithm, direct position determination (DPD) algorithms proposed in recent years do not need to calculate the position step-by-step. Improved positioning performance can be obtained using the signal directly to estimate the position of the target [20-22]. Developing the study of DPD algorithms is more valuable than that of the two-step algorithm.

The DPD algorithm has been extensively investigated in the past decade because of its excellent performance. Weiss first expounded the principle of DPD algorithms and proposed the maximum likelihood (ML) estimation-based method $[23,24]$. To reduce the computational load generated by ML estimation, subspace data fusion- (SDF-) [25] and minimum variance distortionless response-based DPD algorithms [26] are proposed to decrease the dimension of the parameters to be estimated in order to mitigate the computation cost. As the DPD algorithm directly uses array outputs to localize targets, certain signal properties can be exploited to improve localization performance. Thus, the DPD algorithm based on the constant modulus, cyclostationary, and orthogonal frequency division multiplexing properties of the received signals achieve good localization performance [27-29]. Bar proposed the CML-DPD algorithm for CD sources based on the generalized covariance matching method to improve the localization accuracy of CD sources [30]. Although the algorithm outperforms conventional two-step methods in terms of the localization accuracy of CD sources, it is not practically useful because of its huge computational complexity. Moreover, localization performance still has room for improvement because signal characteristics are unused.

In summary, although the two-step localization method for $\mathrm{CD}$ sources improves the parameter estimation accuracy using the noncircular signal features, it cannot achieve optimal performance because of its limitations. In addition, the existing DPD algorithm designed for CD sources do not utilize signal characteristics and the computation cost is
TABLE 1: Mathematical notation explanation.

\begin{tabular}{ll}
\hline Notation & Explanation \\
\hline$\{\bullet\}^{\mathrm{T}},\{\bullet\}^{\mathrm{H}},\{\bullet\}^{\prime}$ & $\begin{array}{l}\text { Represent the transpose, conjugate transpose, and } \\
\text { conjugate, respectively; }\end{array}$ \\
$\operatorname{diag}\{\bullet\}$ & $\begin{array}{l}\text { Indicates the diagonal matrix whose diagonal is a } \\
\text { vector; }\end{array}$ \\
blkdiag $\{\bullet\}$ & $\begin{array}{l}\text { Represents the block-diagonal matrix formed } \\
\text { from the matrices or vectors; }\end{array}$ \\
$\|\bullet\|$ & $\begin{array}{l}\text { Euclidean norm of the matrix; } \\
\text { E[ }[\bullet]\end{array}$ \\
$\mathbf{I}, \mathbf{O}$ & $\begin{array}{l}\text { Signifies the expectation; } \\
\text { Denote the identity matrix and zero matrix, }\end{array}$ \\
$\operatorname{tr}\{\bullet\}$ & $\begin{array}{l}\text { Respectively; } \\
\text { Represents the trace; }\end{array}$ \\
\hline
\end{tabular}

high, such that localization performance should be improved. Therefore, this study presents a novel DPD algorithm that profits from the characteristics of noncircular signals to achieve improved positioning accuracy of $\mathrm{CD}$ sources. An extended SDF method is proposed to reduce the computational load to improve the practical value of the algorithm. The contributions of this study can be summarized as follows.

(1) The proposed DPD model for multiple CD sources fully employs the characteristics of noncircular signals, thereby extending the virtual array aperture of the receivers to improve the localization accuracy and number of detectable sources. To reduce computation cost, an extended SDF method is developed, which requires only low-dimensional optimization, resulting in low complexity and high efficiency.

(2) We derive the closed-form expression of the CRLB on the position estimation variance for noncircular signals to present a benchmark of the highest localization accuracy that the DPD method can reach. In the simulation part, the localization performance of the proposed algorithm can be proven to be close to the CRLB, which shows the effectiveness of our method.

This paper is organized as follows: Section 2 lists the notations used throughout the study. Section 3 describes the signal model for CD sources and formulates the problem. Section 4 derives the extended SDF-DPD estimator based on the characteristics of noncircular signals and analyzes the computation cost. Section 5 derives the closed-form expression of the CRLB on the position estimation variance. Section 6 presents several numerical simulations to validate the accuracy and reliability of the theoretical analysis. Finally, Section 7 concludes the study.

\section{Notations}

The notations used throughout this study are listed in Table 1.

\section{System Model and Problem Formulation}

In this study, the transmitter is assumed to be a far-field narrowband source, and we only consider the localization of $\mathrm{CD}$ sources based on the DOA information. $L$ receivers and $P$ transmitters, which are all stationary, are employed. 
All receivers equipped with a uniform linear antenna array are synchronized. The array is composed of $M$ sensors with the first sensor located at $\mathbf{u}_{l}=\left(u_{l, x}, u_{l, y}\right), l=1,2, \ldots, L$, and the adjacent sensors are spaced at $d_{m}$. The transmitters are located at $\mathbf{o}_{p}=\left(o_{p, x}, o_{p, y}\right), p=1,2, \ldots, P$. The signals of different transmitters are independent of each other. At the $l$ th receiver, the received signal can be expressed as [14]

$$
\begin{aligned}
& \mathbf{r}_{l}(t)=\sum_{p=1}^{P} \int_{-\pi}^{\pi} \mathbf{a}_{l}\left(\phi_{l, p}\left(\mathbf{o}_{p}\right)\right) \\
& \cdot x_{p}\left(\phi_{l, p}\left(\mathbf{o}_{p}\right)-\theta_{l, p}\left(\mathbf{o}_{p}\right), t\right) d \phi_{l, p}\left(\mathbf{o}_{p}\right) \\
& +\mathbf{w}_{l}(t), \quad 0 \leq t \leq T,
\end{aligned}
$$

where $\mathbf{a}_{l}\left(\phi_{l, p}\left(\mathbf{o}_{p}\right)\right)$ is the steering vector of the $p$ th transmitter, $x_{p}\left(\phi_{l, p}\left(\mathbf{o}_{p}\right)-\theta_{l, p}\left(\mathbf{o}_{p}\right), t\right)$ is the angular density signal, and $\mathbf{w}_{l}(t)$ is the statistically independent complex Gaussian noise. $\theta_{l, p}\left(\mathbf{o}_{p}\right)$ is the central direction of arrival as shown in

$$
\theta_{l, p}\left(\mathbf{o}_{p}\right)=\arctan \frac{o_{p, y}-u_{l, y}}{o_{p, x}-u_{l, x}} .
$$

For the CD sources, the angular density signal can be expressed as

$$
\begin{aligned}
& x_{p}\left(\phi_{l, p}\left(\mathbf{o}_{p}\right)-\theta_{l, p}\left(\mathbf{o}_{p}\right), t\right) \\
& =x_{p}(t) g_{p}\left(\phi_{l, p}\left(\mathbf{o}_{p}\right)-\theta_{l, p}\left(\mathbf{o}_{p}\right)\right),
\end{aligned}
$$

where $g_{p}\left(\phi_{l, p}\left(\mathbf{o}_{p}\right)-\theta_{l, p}\left(\mathbf{o}_{p}\right)\right)$ is the deterministic distributed function with the symmetric center of $\theta_{l, p}\left(\mathbf{o}_{p}\right)$ satisfying the following condition:

$$
\int_{-\pi}^{\pi} g_{p}\left(\phi_{l, p}\left(\mathbf{o}_{p}\right)-\theta_{l, p}\left(\mathbf{o}_{p}\right)\right) d \phi_{l, p}\left(\mathbf{o}_{p}\right)=1 .
$$

We let $\mathbf{b}_{l, p}\left(\mathbf{o}_{p}\right)$ be the modified steering vector which can be expressed as

$$
\begin{aligned}
& \mathbf{b}_{l, p}\left(\mathbf{o}_{p}\right)=\int_{-\pi}^{\pi} \mathbf{a}_{l, p}\left(\phi_{l, p}\left(\mathbf{o}_{p}\right)\right) \\
& \cdot g_{p}\left(\phi_{l, p}\left(\mathbf{o}_{p}\right)-\theta_{l, p}\left(\mathbf{o}_{p}\right)\right) d \phi_{l, p}\left(\mathbf{o}_{p}\right) .
\end{aligned}
$$

Then the observed signal can be sampled as

$$
\mathbf{r}_{l}[n]=\sum_{p=1}^{P} \mathbf{b}_{l, p}\left(\mathbf{o}_{p}\right) x_{p}[n]+\mathbf{w}_{l}[n], \quad n=1,2, \ldots, N
$$

where $x_{p}[n]$ is the $n$th samples of $x_{p}, \mathbf{r}_{l}[n]$ is the $n$th samples of $\mathbf{r}_{l}$, and $N$ is the number of samples. We let $\mathbf{x}=$ $\left[x_{1}, x_{2}, \ldots, x_{P}\right]^{\mathrm{T}}$ and $\mathbf{B}_{l}(\widetilde{\mathbf{o}})=\left[\mathbf{b}_{l, 1}\left(\mathbf{o}_{1}\right), \mathbf{b}_{l, 2}\left(\mathbf{o}_{2}\right), \ldots, \mathbf{b}_{l, P}\left(\mathbf{o}_{P}\right)\right]$, where $\widetilde{\mathbf{o}}=\left[\mathbf{o}_{1}^{\mathrm{T}}, \mathbf{o}_{2}^{\mathrm{T}}, \ldots, \mathbf{o}_{P}^{\mathrm{T}}\right]^{\mathrm{T}}$ denotes the position vector of all transmitters. The received signal $\mathbf{r}_{l}$ is then expressed as

$$
\mathbf{r}_{l}=\mathbf{B}_{l}(\widetilde{\mathbf{o}}) \mathbf{x}+\mathbf{w}_{l}
$$

Based on the received signal $\mathbf{r}_{l}$ according to (7), the problem addressed here is to estimate the source positions directly without explicitly computing the DOAs. The subsequent section will describe the novel DPD algorithm in detail.

\section{DPD Algorithm of CD Noncircular Sources}

4.1. NCSDF-DPD Algorithm. Based on the system model proposed in Section 3, $\mathbf{b}_{l, p}\left(\mathbf{o}_{p}\right)$ is closely related to the deterministic distributed function $g_{p}\left(\phi_{l, p}\left(\mathbf{o}_{p}\right)-\theta_{l, p}\left(\mathbf{o}_{p}\right)\right)$. According to (6), when $g_{p}\left(\phi_{l, p}\left(\mathbf{o}_{p}\right)-\theta_{l, p}\left(\mathbf{o}_{p}\right)\right)=\delta\left(\phi_{l, p}\left(\mathbf{o}_{p}\right)-\right.$ $\left.\theta_{l, p}\left(\mathbf{o}_{p}\right)\right), \mathbf{b}_{l, p}\left(\mathbf{o}_{p}\right)=\mathbf{a}_{l, p}\left(\theta_{l, p}\left(\mathbf{o}_{p}\right)\right)$ can be obtained, which is the same as that of the point source model. When $g_{p}\left(\phi_{l, p}\left(\mathbf{o}_{p}\right)-\theta_{l, p}\left(\mathbf{o}_{p}\right)\right)$ is a complex function, obtaining the analytical expression of $\mathbf{b}_{l, p}\left(\mathbf{o}_{p}\right)$ is difficult. Thus, the proposed algorithm is analyzed using the Gaussian angle of arrival (GAA) channel model as an example, similar to that in [30], to discuss the localization performance conveniently. The process employed in this study can be used as reference for the analysis of the performance of other distributed functions.

Based on the GAA channel model, the distribution of the angle perturbations generated by the scatterers is Gaussian with the mean of $\theta_{l, p}\left(\mathbf{o}_{p}\right)$ and the standard deviation of $\sigma_{\theta, l, p}$, such that the $m$ th element of $\mathbf{b}_{l, p}\left(\mathbf{o}_{p}\right)$ can be expressed as

$$
\begin{aligned}
& {\left[\mathbf{b}_{l, p}\left(\mathbf{o}_{p}\right)\right]_{m}} \\
& =\int_{-\pi}^{\pi} \exp \left(-j 2 \pi(m-1) d_{m} \cos \left(\phi_{l, p}\left(\mathbf{o}_{p}\right)\right)\right) \\
& \quad \times \frac{1}{\sqrt{2 \pi} \beta_{\omega, p}} \\
& \quad \cdot \exp \left(\frac{\left(\phi_{l, p}\left(\mathbf{o}_{p}\right)-\theta_{l, p}\left(\mathbf{o}_{p}\right)\right)^{2}}{2 \beta_{\omega, p}^{2}}\right) d \phi_{l}\left(\mathbf{o}_{p}\right),
\end{aligned}
$$

where $\left[\mathbf{b}_{l, p}\left(\mathbf{o}_{p}\right)\right]_{m}$ denotes the $m$ th element of $\mathbf{b}_{l, p}\left(\mathbf{o}_{p}\right) \cdot \beta_{\omega, p}$ is given by

$$
\beta_{\omega, p}=2 \pi d_{m} \sigma_{\theta, l, p} \sin \left(\theta_{l, p}\left(\mathbf{o}_{p}\right)\right) .
$$

In general, the distribution of the scatterers around the transmitter is assumed to be circular symmetric, and the variance of the distribution radius is set as $\varepsilon_{p}$. Thus $\sigma_{\theta, l, p}$ can be approximated as [30]

$$
\sigma_{\theta, l, p} \approx \frac{\varepsilon_{p}}{d_{l, p}}
$$

where $d_{l, p}$ is the distance between the $p$ th transmitter and the $l$ th receiver. We then have the simplified form of (8) as follows [12]:

$$
\mathbf{b}_{l, p}\left(\mathbf{o}_{p}, \varepsilon_{p}\right)=\mathbf{A}_{l, p}\left(\mathbf{o}_{p}\right) \mathbf{G}_{l, p}\left(\mathbf{o}_{p}, \varepsilon_{p}\right)
$$

where

$$
\begin{aligned}
& \mathbf{A}_{l, p}\left(\mathbf{o}_{p}\right)=\operatorname{diag}\left(\mathbf{a}_{l, p}\left(\theta_{l, p}\left(\mathbf{o}_{p}\right)\right)\right), \\
& \mathbf{G}_{l, p}\left(\mathbf{o}_{p}, \varepsilon_{p}\right) \\
& \quad=\left[1, \exp \left(-\frac{1}{2} \beta_{\omega, p}^{2}\right), \ldots, \exp \left(\frac{-(M-1)^{2} \beta_{\omega, p}^{2}}{2}\right)\right] .
\end{aligned}
$$


Through the previously presented analysis, the analytical expression of $\mathbf{b}_{l, p}\left(\mathbf{o}_{p}\right)$ is obtained. The covariance matrix of $\mathbf{r}_{l}$ is expressed in the following form:

$$
\mathbf{R}_{l}=\mathrm{E}\left[\mathbf{r}_{l} \mathbf{r}_{l}^{\mathrm{H}}\right]=\mathbf{B}_{l}(\widetilde{\mathbf{o}}) \mathbf{R}_{x} \mathbf{B}_{l}^{\mathrm{H}}(\widetilde{\mathbf{o}})+\sigma_{l, w}^{2} \mathbf{I}_{M},
$$

where $\sigma_{l, w}^{2}$ is the noise power, $\mathbf{I}_{M}$ is the $M \times M$ identity matrix, and $\mathbf{R}_{x}=\mathrm{E}\left[\mathbf{x x}^{\mathrm{H}}\right]$ is the covariance matrix of signals. The position information of the transmitters is included in $\mathbf{R}_{l}$. In [30], the CML-DPD method is proposed; however, the signal waveform should be known in advance and the computation cost is high when dealing with multitarget localization. Thus, we propose a novel SDF-DPD algorithm based on the characteristics of noncircular signals to achieve improved positioning accuracy of CD sources.

We briefly describe the statistical properties of noncircular signals. $\mathbf{x}_{p}$ is considered to be circular if $\mathrm{E}\left[\mathbf{x}_{p} \mathbf{x}_{p}^{\mathrm{H}}\right] \neq \mathrm{O}$, $\mathrm{E}\left[\mathbf{x}_{p} \mathbf{x}_{p}^{\mathrm{T}}\right]=\mathrm{O}$, whereas $\mathbf{x}_{p}$ is considered to be noncircular if $\mathrm{E}\left[\mathbf{x}_{p} \mathbf{x}_{p}^{\mathrm{H}}\right] \neq \mathrm{O}, \mathrm{E}\left[\mathbf{x}_{p} \mathbf{x}_{p}^{\mathrm{T}}\right] \neq \mathrm{O}$. Moreover, if $\mathbf{x}_{p}$ is a noncircular signal with zero mean, then its variance and unconjugated variance satisfy the following condition:

$$
\mathrm{E}\left[\mathbf{x}_{p}^{2}\right]=\rho e^{\mathrm{j} \varphi_{p}} \mathrm{E}\left[\left|\mathbf{x}_{p}\right|^{2}\right]
$$

where $0 \leq \rho \leq 1$ is the noncircularity rate and $\varphi_{p}$ is the noncircularity phase. Given that the common signals, such as BPSK, MASK, and AM, are the strict-sense noncircular signals with the maximum noncircularity rate $(\rho=1)$, we only analyze the localization of strict-sense noncircular signals in this study. By substituting $\rho=1$ into (15), the unconjugated covariance matrix of $\mathbf{x}$ can be given as

$$
\widehat{\mathbf{R}}_{\mathbf{x}}=\mathrm{E}\left[\mathbf{x x}^{\mathrm{T}}\right]=\boldsymbol{\Phi}_{\varphi} \mathbf{R}_{\mathbf{x}},
$$

where $\boldsymbol{\Phi}_{\varphi}=\operatorname{diag}\left[e^{j \varphi_{1}}, e^{j \varphi_{2}}, \ldots, e^{j \varphi_{P}}\right]$. The diagonal elements are related to the noncircularity phases. The unconjugated covariance matrix of $\mathbf{r}_{l}$ can then be expressed as

$$
\widehat{\mathbf{R}}_{l}=\mathrm{E}\left[\mathbf{r}_{l} \mathbf{r}_{l}^{\mathrm{T}}\right]=\mathbf{B}_{l}(\widetilde{\mathbf{o}}) \widehat{\mathbf{R}}_{x} \mathbf{B}_{l}^{\mathrm{H}}(\widetilde{\mathbf{o}}) .
$$

We extend the observed signal as $\widetilde{\mathbf{r}}_{l}=\left[\mathbf{r}_{l}^{\mathrm{T}}, \mathbf{r}_{l}^{\mathrm{H}}\right]^{\mathrm{T}}$ to apply the unconjugated property of noncircular signals. Then the covariance matrix of $\widetilde{\mathbf{r}}_{l}$ is structured as

$$
\widetilde{\mathbf{R}}_{l}=\mathrm{E}\left[\widetilde{\mathbf{r}}_{l} \widetilde{\mathbf{r}}_{l}^{\mathrm{H}}\right]=\left[\begin{array}{cc}
\mathbf{R}_{l} & \widehat{\mathbf{R}}_{l} \\
\widehat{\mathbf{R}}_{l}^{\prime} & \mathbf{R}_{l}^{\prime}
\end{array}\right] .
$$

We let

$$
\mathbf{b}_{l, p}^{\mathrm{NC}}\left(\mathbf{o}_{p}, \varepsilon_{p}, \varphi_{p}\right)=\left[\begin{array}{c}
\mathbf{b}_{l, p}\left(\mathbf{o}_{p}, \varepsilon_{p}\right) \\
\mathbf{b}_{l, p}^{\prime}\left(\mathbf{o}_{p}, \varepsilon_{p}\right) e^{-\mathrm{j} \varphi_{p}}
\end{array}\right]
$$

We set $\mathbf{B}_{\mathrm{NC}, l}\left(\widetilde{\mathbf{o}}, \mathbf{c}_{\varepsilon}, \mathbf{g}_{\varphi}\right)=\left[\mathbf{b}_{l, 1}^{\mathrm{NC}}, \mathbf{b}_{l, 2}^{\mathrm{NC}}, \ldots, \mathbf{b}_{l, P}^{\mathrm{NC}}\right]$, where $\mathbf{g}_{\varphi}=$ $\left[\varphi_{1}, \varphi_{2}, \ldots, \varphi_{P}\right]$ and $\mathbf{c}_{\varepsilon}=\left[\varepsilon_{1}, \varepsilon_{2}, \ldots, \varepsilon_{P}\right]$. Thus (18) can be rewritten as

$$
\begin{aligned}
\widetilde{\mathbf{R}}_{l} & =\mathrm{E}\left[\widetilde{\mathbf{r}}_{l} \widetilde{\mathbf{r}}_{l}^{\mathrm{H}}\right] \\
& =\mathbf{B}_{\mathrm{NC}, l}\left(\widetilde{\mathbf{o}}, \mathbf{c}_{\varepsilon}, \mathbf{g}_{\varphi}\right) \mathbf{R}_{x} \mathbf{B}_{\mathrm{NC}, l}^{\mathrm{H}}\left(\widetilde{\mathbf{o}}, \mathbf{c}_{\varepsilon}, \mathbf{g}_{\varphi}\right)+\sigma_{l, w}^{2} \mathbf{I}_{2 M} .
\end{aligned}
$$

From the previously presented analysis, the dimension of the virtual steering vector $\mathbf{b}_{l, p}^{\mathrm{NC}}$ is twice as more than that of the original steering vector $\mathbf{b}_{l, p}$. By exploiting the characteristics of noncircular signals, the array aperture can be expanded, which will improve localization accuracy. Given that the virtual steering vectors of each source are independent, the subspace method can be used to reduce the dimension of the parameters to be estimated, which will significantly reduce the computation cost. The subspace decomposition of $\widetilde{\mathbf{R}}_{l}$ can be given by

$$
\widetilde{\mathbf{R}}_{l}=\sum_{i=1}^{P} \lambda_{i} \mu_{i} \mu_{i}^{\mathrm{H}}+\sum_{i=P+1}^{2 M} \lambda_{i} \mu_{i} \mu_{i}^{\mathrm{H}},
$$

where $\lambda_{i}$ is the eigenvalue and $\mu_{i}$ is the eigenvector. Let $\mathbf{U}_{l, x}$ be the signal subspace and $\mathbf{U}_{l, w}$ be the noise subspace. Given that $\mathbf{B}_{\mathrm{NC}, l}$ is orthogonal to $\mathbf{U}_{l, w},\left(\mathbf{b}_{l, p}^{\mathrm{NC}}\right)^{\mathrm{H}} \mathbf{U}_{l, w}=0$ can be obtained. We can then derive the following cost function:

$$
\begin{aligned}
f & \left(\widetilde{\mathbf{o}}, \mathbf{c}_{\varepsilon}, \mathbf{g}_{\varphi}\right) \\
& =\sum_{l=1}^{L}\left(\mathbf{b}_{l}^{\mathrm{NC}}(\mathbf{o}, \varepsilon, \varphi)\right)^{\mathrm{H}} \mathbf{U}_{l, w} \mathbf{U}_{l, w}^{\mathrm{H}}\left(\mathbf{b}_{l}^{\mathrm{NC}}(\mathbf{o}, \varepsilon, \varphi)\right) .
\end{aligned}
$$

The target position can be obtained by determining the minimum of $f\left(\widetilde{\mathbf{o}}, \mathbf{c}_{\varepsilon}, \mathbf{g}_{\varphi}\right)$. In reducing the number of estimated parameters further, we determine that $\mathbf{g}_{\varphi}$ is unrelated to the target position, such that it can be removed [14]. Based on the characteristics of noncircular signals, the following expression can be obtained:

$$
\mathbf{D}_{l}=\mathbf{U}_{l, w} \mathbf{U}_{l, w}^{\mathrm{H}}=\left[\begin{array}{ll}
\mathbf{D}_{l, 1} & \mathbf{D}_{l, 2} \\
\mathbf{D}_{l, 2}^{\prime} & \mathbf{D}_{l, 1}^{\prime}
\end{array}\right],
$$

where $\mathbf{D}_{l, 1}$ and $\mathbf{D}_{l, 2}$ have the same dimension. Then (22) can be rewritten as

$$
\tilde{f}\left(\widetilde{\mathbf{o}}, \mathbf{c}_{\varepsilon}\right)=\sum_{l=1}^{L}\left(\mathbf{Q}_{l, 1} \mathbf{Q}_{l, 1}^{\prime}-\mathbf{Q}_{l, 2} \mathbf{Q}_{l, 2}^{\prime}\right),
$$

where

$$
\begin{aligned}
& \mathbf{Q}_{l, 1}=\mathbf{b}_{l}^{\mathrm{H}}(\mathbf{o}, \varepsilon) \mathbf{D}_{l, 1} \mathbf{b}_{l}(\mathbf{o}, \varepsilon) \\
& \mathbf{Q}_{l, 2}=\mathbf{b}_{l}^{\mathrm{H}}(\mathbf{o}, \varepsilon) \mathbf{D}_{l, 2} \mathbf{b}_{l}^{\prime}(\mathbf{o}, \varepsilon) .
\end{aligned}
$$

Thus, the position parameters of the CD sources can be obtained using the following equation:

$$
\left(\widetilde{\widetilde{\mathbf{o}}}, \widehat{\mathbf{c}}_{\varepsilon}\right)=\underset{\left(\widetilde{\mathbf{o}}, \mathbf{c}_{\varepsilon}\right)}{\arg \min } \sum_{l=1}^{L}\left(\mathbf{Q}_{l, 1} \mathbf{Q}_{l, 1}^{\prime}-\mathbf{Q}_{l, 2} \mathbf{Q}_{l, 2}^{\prime}\right) .
$$

In summary, compared with the CML-DPD algorithm proposed in [30], the proposed algorithm in this study reduces the dimension of the estimated parameters of multiple CD sources by the SDF method, which can significantly decrease computational load. Moreover, by applying the characteristics of noncircular signals, the signal subspace is 


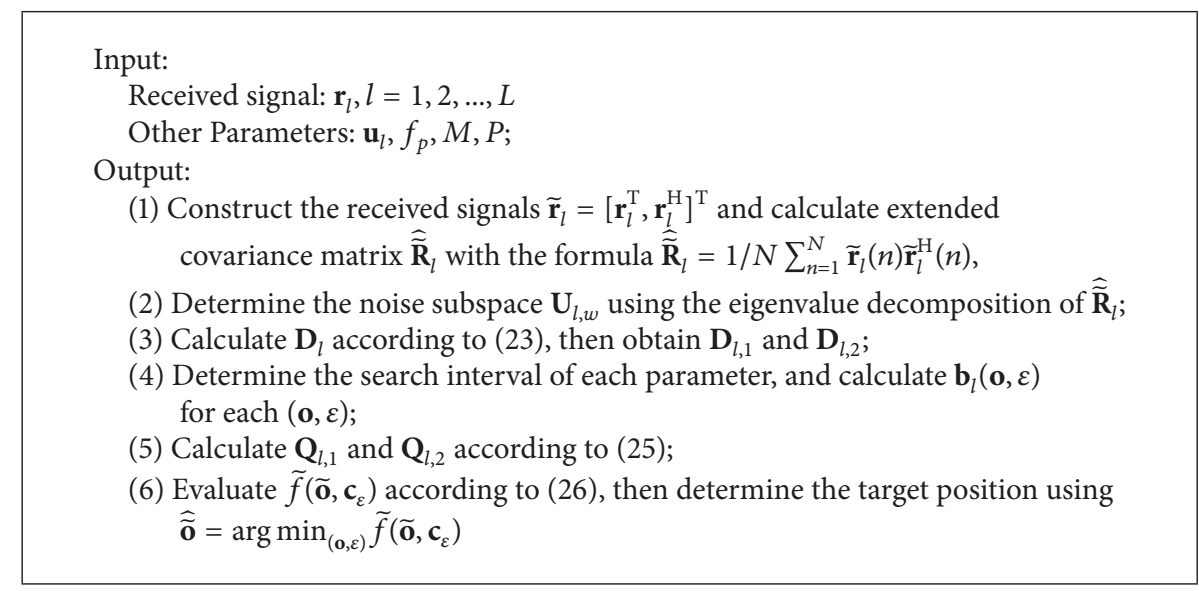

Algorithm 1: NCSDF-DPD algorithm.

TABLE 2: Computational complexity.

\begin{tabular}{|c|c|c|c|}
\hline Algorithm & parameter dimension & Main computations & Amount of computation \\
\hline \multirow{5}{*}{ CML-DPD } & \multirow{5}{*}{$P K$} & (1) $\mathbf{R}_{l}^{-1}$ & $O\left(L M^{3}\right)$ \\
\hline & & (2) $\mathbf{X}=\mathbf{R}_{l}^{-1} \widehat{\mathbf{R}}_{l}$ & $O\left(L M^{3}\right)$ \\
\hline & & (3) $\operatorname{tr}\{\mathbf{X}\}$ & $O\left(4 L M^{3}\right)$ \\
\hline & & $(4)|\mathbf{R}|$ & $O\left(4 L M^{3}\right)$ \\
\hline & & Total: & $O\left(10 L M^{3} J^{P K}\right)$ \\
\hline \multirow{3}{*}{ NCSDF-DPD } & \multirow{3}{*}{$K$} & (1) $\mathbf{Q}_{l, 1}, \mathbf{Q}_{l, 2}$ & $O\left(2 L\left[M^{2}+M\right]\right)$ \\
\hline & & (2) $\tilde{f}$ & $O\left(2 L M^{3}\right)$ \\
\hline & & Total: & $O\left(2 L\left[M^{3}+M^{2}+M\right] J^{K}\right)$ \\
\hline
\end{tabular}

enhanced and the noise subspace is extended, which will improve localization accuracy and the number of detectable CD sources. The simulation experiments will further verify the performance of the algorithm. Given that this algorithm combines the noncircular signal features and SDF method, it is called the NCSDF-DPD algorithm. To present the algorithm better, the NCSDF-DPD algorithm based on the principle derived in this section can be implemented as in Algorithm 1.

4.2. Computational Complexity Analysis. Table 2 lists the main formulas and the amount of calculation in the NCSDFDPD and CML-DPD algorithms [30] to illustrate the difference of the computation cost between the two algorithms. The exhaustive search method is used in the two algorithms for reasonable comparison and analysis because of the large gap in the computational load of different search methods. In Table 2, the number of grids divided on each parameter is $J$, and the parameter dimension that needs to be estimated for one source is $K$.

The comparison results shown in Table 2 indicate that the CML-DPD algorithm requires a larger amount of calculation than the NCSDF-DPD algorithm because the dimension of the estimated parameters of the CML-DPD algorithm is larger than that of the NCSDF-DPD algorithm. However, the computational load of the NCSDF-DPD algorithm is significantly decreased, as expected. Moreover, through several fast calculation methods, such as genetic algorithm, particle filtering method, and expectation maximization algorithm, the computation cost will be further reduced to improve the localization performance.

\section{CRLB for the Position of CD Noncircular Sources}

The CRLB is a valid benchmark used to evaluate the best localization performance that a method can reach. This section derives the closed-form expression of the CRLB on the position estimation variance for CD noncircular sources. The parameters that should be estimated can be expressed as

$$
\mathbf{y}=\left[\widetilde{\mathbf{o}}^{\mathrm{T}}, \mathbf{c}_{\varepsilon}, \mathbf{g}_{\varphi}, \mathbf{z}_{x}, \mathbf{v}_{\sigma}\right]^{T},
$$

where

$$
\begin{aligned}
\widetilde{\mathbf{o}}^{\mathrm{T}} & =\left[o_{1, x}, o_{1, y}, \ldots, o_{p, x}, o_{, y}, \ldots, o_{P, x}, o_{P, y}\right] \\
\mathbf{c}_{\varepsilon} & =\left[\varepsilon_{1}, \varepsilon_{2}, \ldots, \varepsilon_{P}\right] \\
\mathbf{g}_{\varphi} & =\left[\varphi_{1}, \varphi_{2}, \ldots, \varphi_{P}\right] \\
\mathbf{z}_{x} & =\left[z_{1}, z_{2}, \ldots, z_{P}\right] \\
\mathbf{v}_{\sigma} & =\left[\sigma_{1, w}^{2}, \sigma_{2, w}^{2}, \ldots, \sigma_{L, w}^{2}\right]
\end{aligned}
$$


$\mathbf{z}_{x}$ is the vector of signal power, and $\mathbf{v}_{\sigma}$ is the vector of noise power. The Fisher information matrix (FIM) of unknown vector $\mathbf{y}$ is given by

$$
\begin{aligned}
\mathbf{J}_{i, j}=\sum_{l=1}^{L} \operatorname{tr}\left\{\widetilde{\mathbf{R}}_{l}^{-1} \frac{\partial \widetilde{\mathbf{R}}_{l}}{\partial \mathbf{y}_{i}} \widetilde{\mathbf{R}}_{l}^{-1} \frac{\partial \widetilde{\mathbf{R}}_{l}}{\partial \mathbf{y}_{j}}\right\} & \\
& i, j=1,2, \ldots, 5 P+L,
\end{aligned}
$$

where $\mathbf{y}_{i}$ and $\mathbf{y}_{j}$ denote the $i$ th and $j$ th elements of $\mathbf{y}$, respectively, and $\mathbf{J}_{i, j}$ signifies the $(i, j)$ th element of $\mathbf{J}$. According to (20), $\partial \widetilde{\mathbf{R}}_{l} / \partial \mathbf{o}_{p}$ is the partial derivative with respect to $\mathbf{o}_{p}$, which can be obtained using the following equation:

$$
\frac{\partial \widetilde{\mathbf{R}}_{l}}{\partial \mathbf{o}_{p}}=\frac{\partial \mathbf{B}_{\mathrm{NC}, l}}{\partial \mathbf{o}_{p}} \mathbf{R}_{x} \mathbf{B}_{\mathrm{NC}, l}^{\mathrm{H}}+\mathbf{B}_{\mathrm{NC}, l} \mathbf{R}_{x}\left(\frac{\partial \mathbf{B}_{\mathrm{NC}, l}}{\partial \mathbf{o}_{p}}\right)^{\mathrm{H}} .
$$

We let $\left[\mathbf{B}_{\mathrm{NC}, l}\right]_{m, k}, m=1,2, \ldots, M, k=1,2, \ldots, P$ denote the $(m, k)$ th element of $\mathbf{B}_{\mathrm{NC}, l}$, which can be expressed as

$$
\begin{aligned}
{\left[\mathbf{B}_{\mathrm{NC}, l}\right]_{m, k}=} & \exp \left(-\mathrm{j} 2 \pi(m-1) d_{m} \cos \left(\theta_{l, k}\left(\mathbf{o}_{k}\right)\right)\right) \\
& \times \exp \left(-(m-1)^{2} \frac{\beta_{\omega, k}^{2}}{2}\right) .
\end{aligned}
$$

We then derive the following expression:

$$
\begin{gathered}
\frac{\partial\left[\mathbf{B}_{\mathrm{NC}, l}\right]_{m, k}}{\partial o_{p, x}} \\
=-\mathrm{j} 2 \pi(m-1) d_{m} \frac{\partial \cos \left(\theta_{l, k}\left(\mathbf{o}_{k}\right)\right)}{\partial o_{p, x}}\left[\mathbf{B}_{\mathrm{NC}, l}\right]_{m, k} \\
\quad-(m-1)^{2} \beta_{\omega, k} \frac{\partial \beta_{\omega, k}}{\partial o_{p, x}}\left[\mathbf{B}_{\mathrm{NC}, l}\right]_{m, k} \\
\frac{\partial\left[\mathbf{B}_{\mathrm{NC}, l}\right]_{m, k}}{\partial o_{p, y}} \\
=-\mathrm{j} 2 \pi(m-1) d_{m} \frac{\partial \cos \left(\theta_{l, k}\left(\mathbf{o}_{k}\right)\right)}{\partial o_{p, y}}\left[\mathbf{B}_{\mathrm{NC}, l}\right]_{m, k} \\
\left.\quad-(m-1)^{2} \beta_{\omega, k} \frac{\partial \beta_{\omega, k}\left[\mathbf{B}_{\mathrm{NC}, l}\right]_{m, k}}{\partial o_{p, y}}\right)^{\partial\left[\mathbf{B}_{\mathrm{NC}, l}\right]_{2 M-m+1, k}} \\
\frac{\partial o_{p, x}}{\left.\partial \mathbf{B}_{\mathrm{NC}, l}\right]_{2 M-m+1, k}}=\left(\frac{\partial\left[\mathbf{B}_{\mathrm{NC}, l}\right]_{m, k}}{\partial o_{p, x}}\right)^{\prime} \exp \left(-\mathrm{j} \varphi_{p}\right) \\
\left.\frac{\left.\partial \mathbf{B}_{\mathrm{NC}, l}\right]_{m, k}}{\partial o_{p, y}}\right)^{\prime} \exp \left(-\mathrm{j} \varphi_{p}\right) .
\end{gathered}
$$

When $k \neq p, \partial\left[\mathbf{B}_{\mathrm{NC}, l}\right]_{m, k} / \partial o_{p, x}=0$, and $\partial\left[\mathbf{B}_{\mathrm{NC}, l}\right]_{m, k} / \partial o_{p, y}=$ 0 . When $k=p$, we derive the following expression:

$$
\begin{aligned}
& \frac{\partial \cos \left(\theta_{l, k}\left(\mathbf{o}_{k}\right)\right)}{\partial o_{p, x}}=\frac{\left(o_{p, y}-u_{l, y}\right)^{2}}{d_{l, p}^{3}} \\
& \frac{\partial \cos \left(\theta_{l, k}\left(\mathbf{o}_{k}\right)\right)}{\partial o_{p, y}}=-\frac{\left(o_{p, x}-u_{l, x}\right)\left(o_{p, y}-u_{l, y}\right)}{d_{l, p}^{3}},
\end{aligned}
$$

$$
\begin{aligned}
& \frac{\partial \beta_{\omega, k}}{\partial o_{p, x}}=-2 \pi \varepsilon_{p} \frac{\left(o_{p, x}-u_{l, x}\right)\left(o_{p, y}-u_{l, y}\right)}{d_{l, p}^{4}} \\
& \frac{\partial \beta_{\omega, k}}{\partial o_{p, y}}=\frac{\pi \varepsilon_{p}}{d_{l, p}^{2}}-2 \pi \varepsilon_{p} \frac{\left(o_{p, y}-u_{l, y}\right)^{2}}{d_{l, p}^{4}} .
\end{aligned}
$$

$\partial \widetilde{\mathbf{R}}_{l} / \partial \varepsilon_{p}$ is the partial derivative with respect to $\varepsilon_{p}$, which can be obtained by

$$
\frac{\partial \widetilde{\mathbf{R}}_{l}}{\partial \varepsilon_{p}}=\frac{\partial \mathbf{B}_{\mathrm{NC}, l}}{\partial \varepsilon_{p}} \mathbf{R}_{x} \mathbf{B}_{\mathrm{NC}, l}^{\mathrm{H}}+\mathbf{B}_{\mathrm{NC}, l} \mathbf{R}_{x}\left(\frac{\partial \mathbf{B}_{\mathrm{NC}, l}}{\partial \varepsilon_{p}}\right)^{\mathrm{H}},
$$

where

$$
\begin{aligned}
\frac{\partial\left[\mathbf{B}_{\mathrm{NC}, l}\right]_{m, k}}{\partial \varepsilon_{p}} & =-(m-1)^{2} \beta_{\omega, k} \frac{\partial \beta_{\omega, k}}{\partial \varepsilon_{p}}\left[\mathbf{B}_{\mathrm{NC}, l}\right]_{m, k} \\
\frac{\partial\left[\mathbf{B}_{\mathrm{NC}, l}\right]_{2 M-m+1, k}}{\partial \varepsilon_{p}} & =\left(\frac{\partial\left[\mathbf{B}_{\mathrm{NC}, l}\right]_{m, k}}{\partial \varepsilon_{p}}\right)^{\prime} \exp \left(-\mathrm{j} \varphi_{p}\right), \\
\frac{\partial\left[\mathbf{B}_{\mathrm{NC}, l}\right]_{m, k}}{\partial \varepsilon_{p}} & =0 \\
\frac{\partial\left[\mathbf{B}_{\mathrm{NC}, l}\right]_{2 M-m+1, k}}{\partial \varepsilon_{p}} & =0,
\end{aligned}
$$

and where $\partial \beta_{\omega, k} / \partial \varepsilon_{p}=2 \pi d_{m} \sin \left(\theta_{l, p}\left(\mathbf{o}_{p}\right)\right) / d_{l, p}$.

$\partial \widetilde{\mathbf{R}}_{l} / \partial \varphi_{p}$ is the partial derivative with respect to $\varphi_{p}$, which can be obtained by

$$
\frac{\partial \widetilde{\mathbf{R}}_{l}}{\partial \varphi_{p}}=\frac{\partial \mathbf{B}_{\mathrm{NC}, l}}{\partial \varphi_{p}} \mathbf{R}_{x} \mathbf{B}_{\mathrm{NC}, l}^{\mathrm{H}}+\mathbf{B}_{\mathrm{NC}, l} \mathbf{R}_{x}\left(\frac{\partial \mathbf{B}_{\mathrm{NC}, l}}{\partial \varphi_{p}}\right)^{\mathrm{H}},
$$

where

$$
\begin{aligned}
& \frac{\partial\left[\mathbf{B}_{\mathrm{NC}, l}\right]_{m, k}}{\partial \varphi_{p}}=0 \\
& \frac{\partial\left[\mathbf{B}_{\mathrm{NC}, l}\right]_{2 M-m+1, k}}{\partial \varphi_{p}}=-\mathrm{j}\left[\mathbf{B}_{\mathrm{NC}, l}\right]_{2 M-m+1, k}, \\
& k=p \\
& \frac{\partial\left[\mathbf{B}_{\mathrm{NC}, l}\right]_{m, k}}{\partial \varphi_{p}}=0 \\
& \frac{\partial\left[\mathbf{B}_{\mathrm{NC}, l}\right]_{2 M-m+1, k}}{\partial \varphi_{p}}=0,
\end{aligned}
$$


The $P$ sources are independent of each other; then there is $\mathbf{R}_{x}=\operatorname{diag}\left\{\mathbf{z}_{x}\right\}$. Thus we obtain the following expression:

$$
\begin{aligned}
\frac{\partial \widetilde{\mathbf{R}}_{l}}{\partial z_{p}} & =\mathbf{B}_{\mathrm{NC}, l} \frac{\partial \mathbf{R}_{x}}{\partial z_{p}} \mathbf{B}_{\mathrm{NC}, l}^{\mathrm{H}}, \\
\frac{\partial \mathbf{R}_{x}}{\partial z_{p}} & =\operatorname{diag}\{[0,0, \ldots, 1, \ldots, 0,0]\},
\end{aligned}
$$

where $\operatorname{diag}\{[0,0, \ldots, 1, \ldots, 0,0]\}$ is the $p$ th element on the diagonal of $\partial \mathbf{R}_{x} / \partial z_{p}$ which is 1 , and the other elements are 0 .

$\partial \widetilde{\mathbf{R}}_{l} / \partial \sigma_{l, w}^{2}$ is the partial derivative with respect to $\sigma_{l, w}^{2}$, which can be obtained as follows:

$$
\frac{\partial \widetilde{\mathbf{R}}_{l}}{\partial \sigma_{l, w}^{2}}=\mathbf{I}_{2 M} .
$$

In conclusion, the partial derivative with respect to any element of $\mathbf{y}$ can be obtained, such that the FIM can be derived according to (29). We then determine the CRLB which is the inverse of $\mathbf{J}$, as follows:

$$
\operatorname{CRLB}=\operatorname{diag}\left\{\mathbf{J}^{-1}\right\} .
$$

The CRLB denotes the best performance that any unbiased estimator can achieve. When the parameter estimation model is determined, the CRLB can effectively verify the effectiveness of one algorithm by comparing it with the CRLB. In this study, the numerical simulation results of the CRLB will be obtained and compared with all algorithms to assess the localization performance of each algorithm.

\section{Simulation Results}

The simulation experiments will be performed in detail in this section to verify the localization performance of the proposed algorithm in Section 4. To analyze the performance differences between the existing algorithms, the experiments will include the following three algorithms:

(1) The NCSDF-DPD algorithm proposed in this study

(2) The DPD algorithm for CD source without using the characteristics of noncircular signals proposed in [30]: but as the ML method is too complex to get the simulation result for multisources, we replace it with SDF method for easy simulation, which has almost the same performance with the ML method (the method is denoted as CSDF-DPD)

(3) The DPD algorithm for point source without using the characteristics of noncircular signals proposed in [25] (represented as SDF-DPD).

Moreover, the CRLBs for the NCSDF-DPD algorithm derived in Section 5 and for the CSDF-DPD algorithm obtained in [30] are presented to make our results as comprehensive as possible.

We assume that a 2D planar geometry has four observation stations located at $\mathbf{u}_{1}=(-1500,-2000) \mathrm{m}, \mathbf{u}_{2}=(-500$, $-2000) \mathrm{m}, \mathbf{u}_{3}=(0,-2000) \mathrm{m}$, and $\mathbf{u}_{4}=(1000,-2000) \mathrm{m}$. Every station is equipped with a uniform linear array (ULA) with $M=3$ sensors. The adjacent sensors are spaced at $d_{m}=0.5 \lambda$, where $\lambda$ is the largest wavelength of all signals. The signal waveforms are generated as narrowband strictsense noncircular signals that are independently Gaussiandistributed. The sampling frequency is $f_{s}=1 \mathrm{MHz}$, and the sampling number is $N=200$. We assume that two near CD sources are located at $\mathbf{o}_{1}=(-1200,0) \mathrm{m}$ and $\mathbf{o}_{2}=(-1000,200) \mathrm{m}$, respectively, to verify the localization accuracy of the proposed algorithm. The variance of the distribution radius is $\mathbf{c}_{\varepsilon}=[280,300]$, and the noncircularity phase is $\mathbf{g}_{\varphi}=[\pi / 3, \pi / 5] \mathrm{rad}$.

The evaluation of the inverse cost functions, that is, $1 / \tilde{f}\left(\widetilde{\mathbf{o}}, \mathbf{c}_{\sigma}\right)$, is conducted under the condition that $\mathrm{SNR}=15 \mathrm{~dB}$ as shown in Figure 1. The $3 \mathrm{D}$ plots and their top views are presented to show the results clearly, wherein the $x$ - and $y$ axes are the position coordinates. Figure 1(a) shows that the position estimations of two sources are seriously mixed up with a large fuzzy region because the SDF-DPD algorithm utilizes the point source model to locate CD sources. Thus obtaining the exact source number and the accurate position estimation is impossible. The results show that the point source model is invalid and cannot be used to solve the localization of CD sources. Figure 1(b) indicates that a sharp peak near the real position is obtained by the CSDF-DPD algorithm, which considers the distribution characteristics of the CD sources to improve localization performance. However, the algorithm cannot distinguish the two sources effectively because they are close to each other, resulting in only one location result with a large deviation. Figure 1(c) shows that, compared with the two other algorithms, the NCSDF-DPD algorithm can clearly distinguish two sources and yield high-accuracy location results by applying the characteristics of noncircular signals. The simulation results indicate that the distribution characteristics of CD sources should be considered in the localization algorithm; otherwise, the positioning accuracy will be poor. By contrast, the localization performance of the proposed algorithm is improved, as expected, which proves the effectiveness of the method that uses noncircular signal features to enhance the positioning accuracy of CD sources.

Given that the dimension of the virtual steering vector is two times more than that of the original steering vector, the array aperture is expanded by exploiting the characteristics of noncircular signals, which improves localization performance compared with that of the CSDF-DPD algorithm under the same array scale. We assumed that the three CD sources are located at $\mathbf{o}_{1}=(-1200,0) \mathrm{m}, \mathbf{o}_{2}=(500,0) \mathrm{m}$, and $\mathbf{o}_{3}=(1000,500) \mathrm{m}$ to verify this performance. The variance of distribution radius is $\boldsymbol{c}_{\varepsilon}=[200,210,220]$, and the noncircularity phases are $\mathbf{g}_{\varphi}=[\pi / 3, \pi / 5, \pi / 4] \mathrm{rad}$. The evaluation of the inverse cost functions of the CSDFDPD and NCSDF-DPD algorithms is obtained under the condition that $\mathrm{SNR}=15 \mathrm{~dB}$, as shown in Figure 2. The results show that the CSDF-DPD algorithm can obtain only two position estimations of three CD sources, with low localization accuracy, whereas the NCSDF-DPD algorithm can derive all the position estimations of three CD sources, with high localization accuracy. The results can be attributed to the CSDF-DPD algorithm only able to locate two sources when the number of array sensors is three, whereas the 

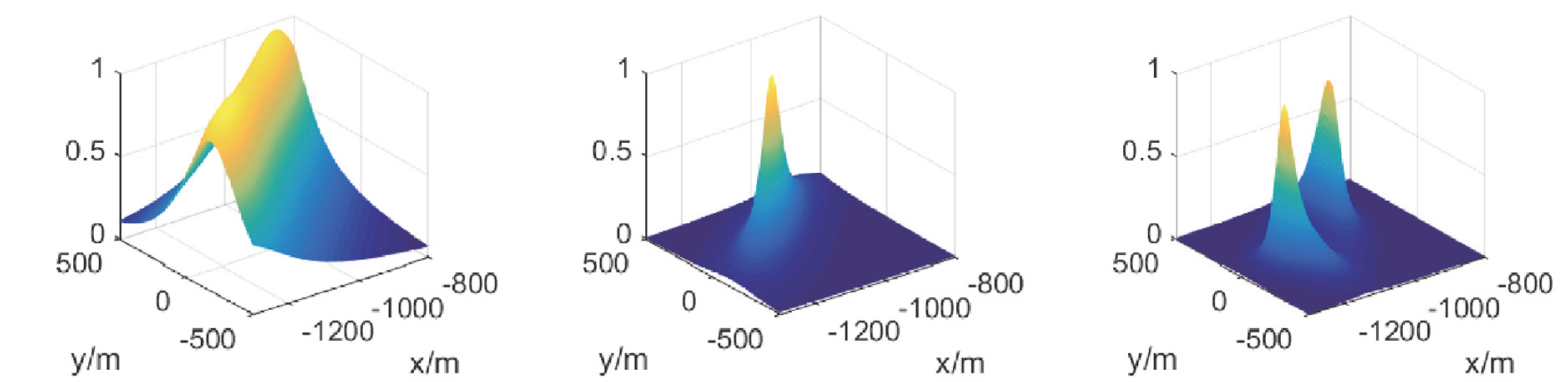

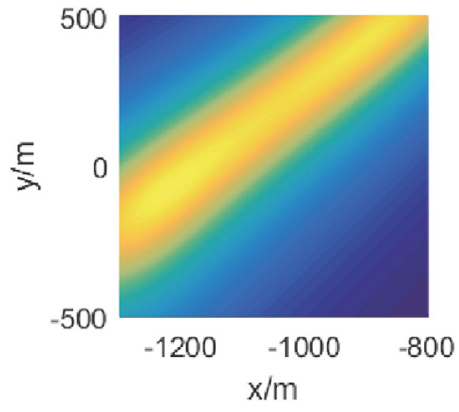

(a)

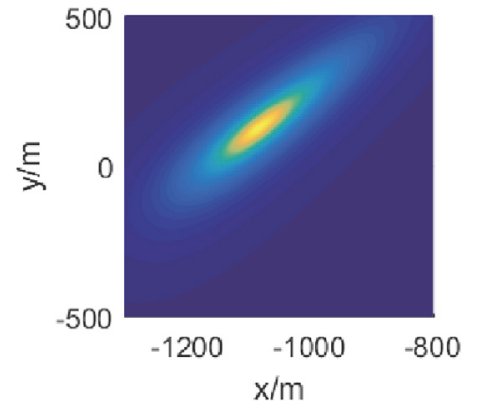

(b)

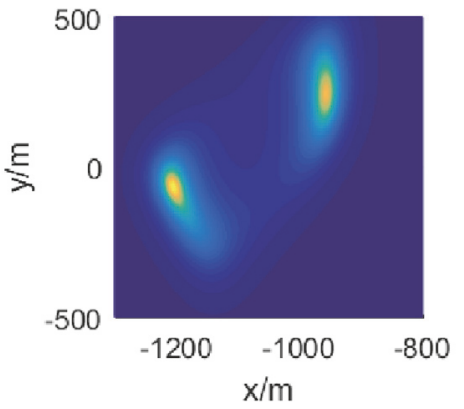

(c)

FIgURE 1: Evaluation of the inverse cost functions of the three algorithms in the scenario where four receivers with three sensors intercept two CD sources. (a) SDF-DPD algorithm, (b) CSDF-DPD algorithm, and (c) NCSDF-DPD algorithm.

NCSDF-DPD algorithm can locate five sources under the same conditions using the characteristics of noncircular signals. The simulation results further verify the effectiveness of the proposed algorithm and indicate that the NCSDFDPD algorithm can achieve better localization performance when the array aperture is small, which enhances the practical value.

Figure 1 shows that the distribution characteristics of CD sources need to be considered in the localization algorithm. We assumed that the two CD sources are located at $\mathbf{o}_{1}=$ $(-1200,0) \mathrm{m}$ and $\mathbf{o}_{2}=(0,0) \mathrm{m}$ to verify the location performance of the three algorithms under different distribution radii. The variances of the distribution radii of the two sources are the same at $[100,250,350]$. Under the condition that SNR $=15 \mathrm{~dB}, K_{c}=100$ independent simulation experiments of the three algorithms are conducted. The cumulative distribution function $(\mathrm{CDF})$ is set as follows:

$$
\operatorname{CDF}\left(w_{e}\right)=\frac{\operatorname{Num}\left(\operatorname{err} \leq w_{e}\right)}{K_{c}},
$$

where $w_{e}$ is the positioning error. The CDFs of the three algorithms are shown in Figure 3. The findings indicate that the positioning performance of the SDF-DPD algorithm worsens with the enlargement of the variance of the distribution radius, which again shows that the distribution of the source adversely affects the performance of conventional point source localization algorithms. The positioning performance of the CSDF-DPD algorithm is improved after considering the distribution characteristics of the sources but is still reduced with the enlargement of the variance of the distribution radius. Compared with the two other algorithms, the NCSDF-DPD algorithm is insensitive to the variance of the distribution radius after using the noncircular signal features to extend the subspace. Thus, with the increase in variance, the localization performance of the NCSDFDPD algorithm does not decline and is better than that of the SDF-DPD and CSDF-DPD algorithms under any distribution radius, which further illustrates the effectiveness of the algorithm.

The root-mean-square error (RMSE) and CRLB are regarded as functions of SNR across 100 Monte Carlo experiments, as shown in Figure 4, to illustrate the localization performance of each algorithm further. The RMSEs of the SDFDPD and CSDF-DPD algorithms are large at low SNR, and their localization performances are nearly the same because noise is the main factor affecting positioning accuracy. However, at high SNR, the RMSE of the CSDF-DPD algorithm can almost reach the CRLB, whereas the SDF-DPD algorithm performs poorly. This performance may be attributed to the distribution characteristics of the sources at this time being the main factors that affect positioning accuracy. Compared with the CSDF-DPD algorithm, although the performance of the NCSDF-DPD algorithm is slightly reduced using the subspace method to decrease the computation cost, its CRLB and RMSE are lower, particularly when the SNR is low. The simulation results again demonstrate the effectiveness of the proposed algorithm because of the use of the characteristics of noncircular signals.

Owing to the expansion of the virtual steering vector, the number of samples required should be decreased. Under 

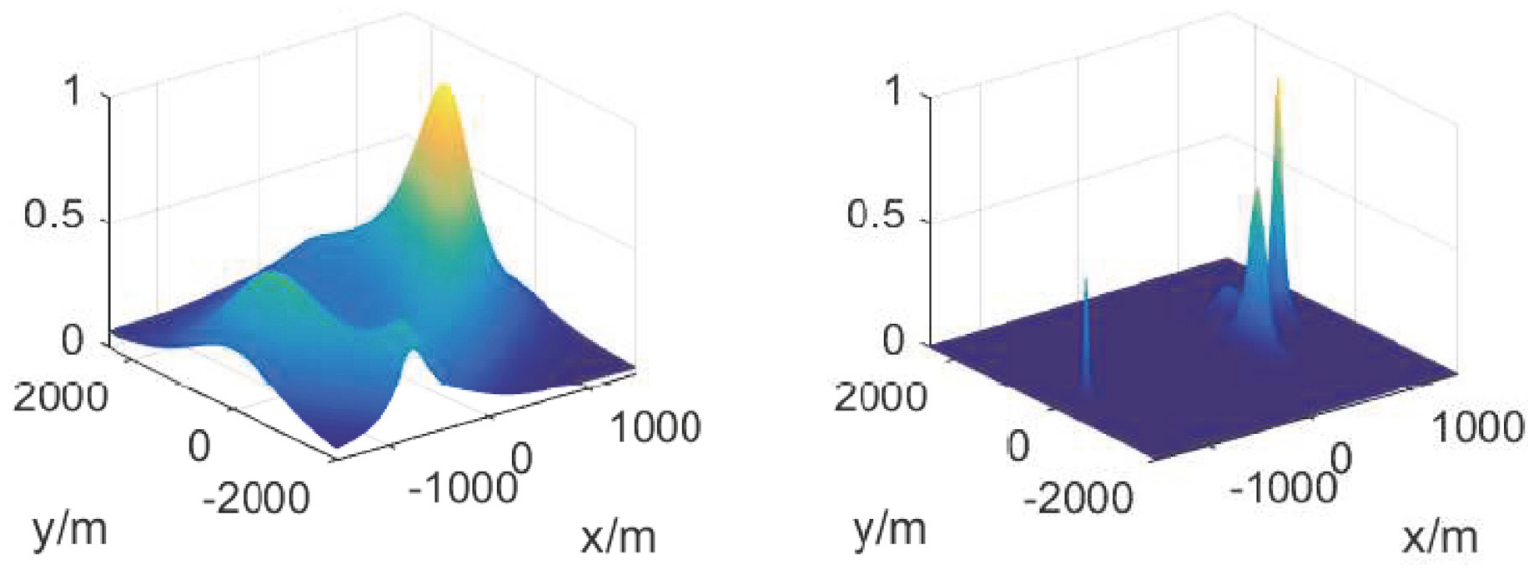

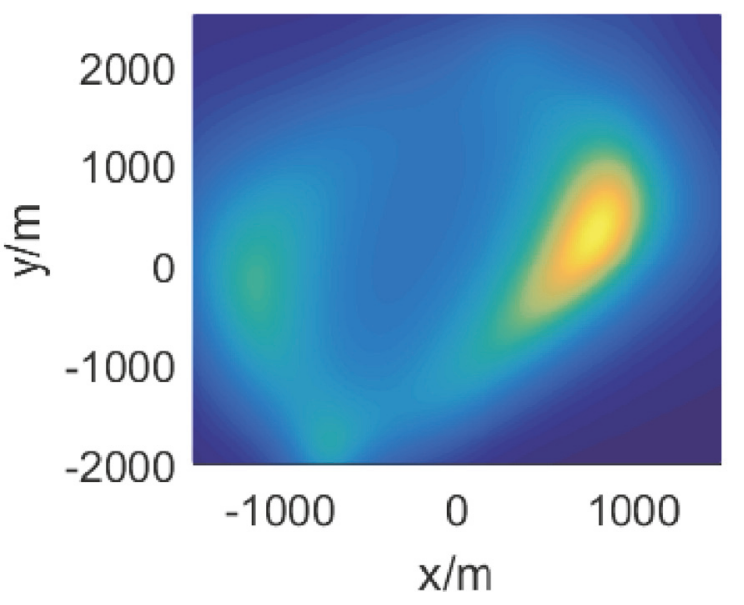

(a)

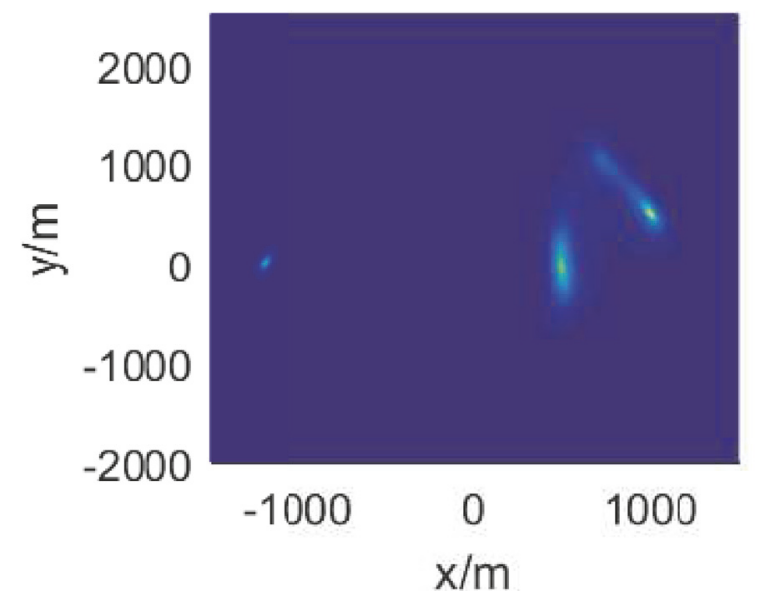

(b)

FIGURE 2: Evaluation of the inverse cost functions of CSDF-DPD and NCSDF-DPD algorithms in the scenario wherein four receivers with three sensors intercept three CD sources. (a) CSDF-DPD algorithm and (b) NCSDF-DPD algorithm.

the condition that $\mathrm{SNR}=5 \mathrm{~dB}$, the influence of the number of samples on the positioning performance is shown in Figure 5. The results indicate that the performance of the SDF-DPD algorithm is not significantly improved with the increase in the number of samples, whereas the RMSE of the NCSDF-DPD algorithm decreases obviously, such that its performance is better than that of the SDF-DPD and CSDFDPD algorithms. Moreover, the NCSDF-DPD algorithm under the condition of small samples can outperform the CSDF-DPD algorithm under the condition of large samples, which indicate that the observation time can be shortened to increase the localization timeliness.

Table 2 lists the main formulas and the amount of calculation in the proposed method and the DPD method in [30]. Now we give the running-times to further illustrate the computation cost of each method. The simulation experiment environment is as follows: Intel Core i7 CPU $2.4 \mathrm{GHz}$ and MATLAB2015. The running-times are regarded as functions of the grids number divided on each parameter. Simulation results are shown in Figure 6 (for single source). We can conclude from the results that, with the increase of grids number, the running-time of the DPD method in [30] increases sharply. However, that of the proposed method in this study increases slowly. Moreover, the more the grids are, the more effective the proposed method is than the DPD method in [30]. So the proposed method is more practical.

\section{Conclusions}

The localization of CD sources is a difficult problem in complex environment. The algorithm proposed in this study is closer to real applications in terms of localization scenarios and signal models. In order to further improve the positioning performance, this paper presents a DPD algorithm with combined the characteristics of noncircular sources and subspace data fusion method. The closed-form expression of CRLB on the position estimation variance for noncircular sources is also derived. In the simulation part, the localization performance of the proposed algorithm can be proven to be better than the existing algorithms, with higher positioning accuracy and lower computation cost. The proposed algorithm exhibits good performance when 

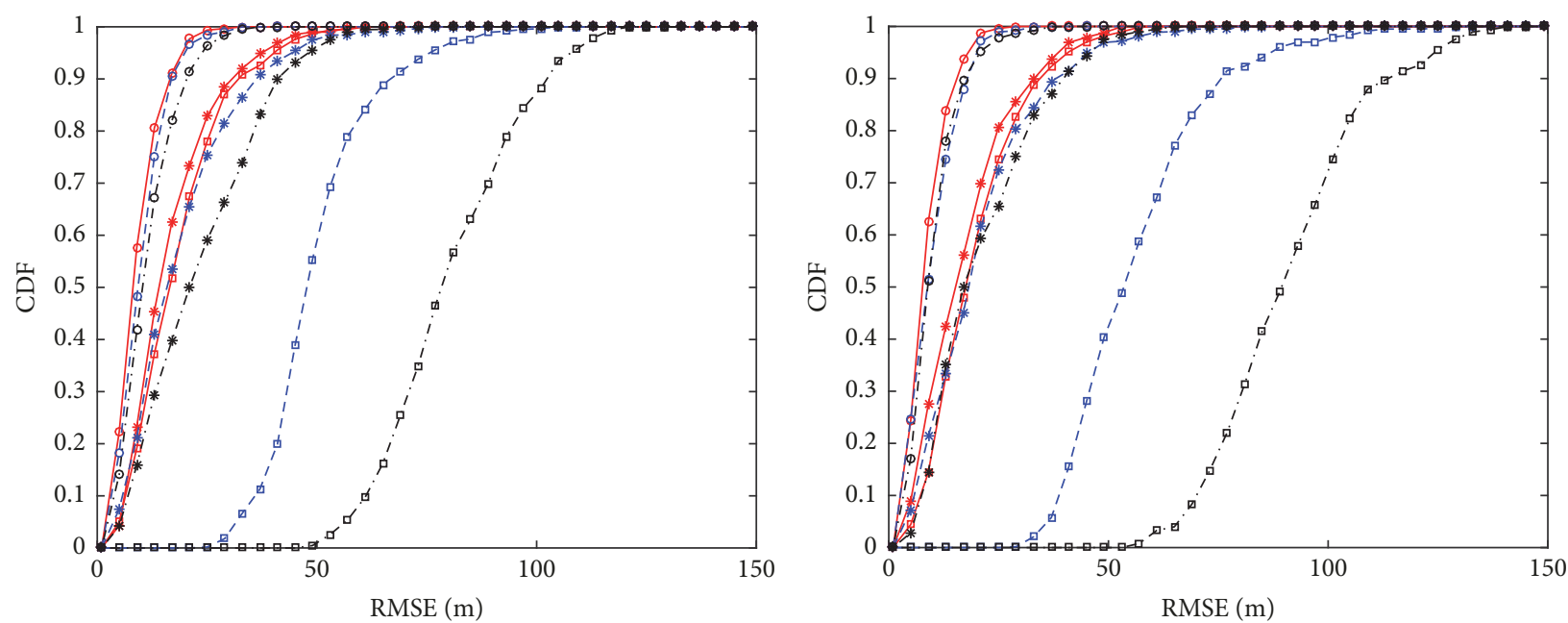

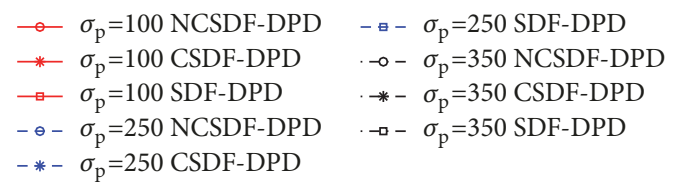

(a) Source 1

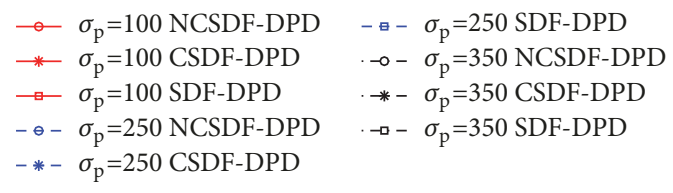

(b) Source 2

Figure 3: The CDF of three algorithms with different distribution radius: (a) source 1; (b) source 2.

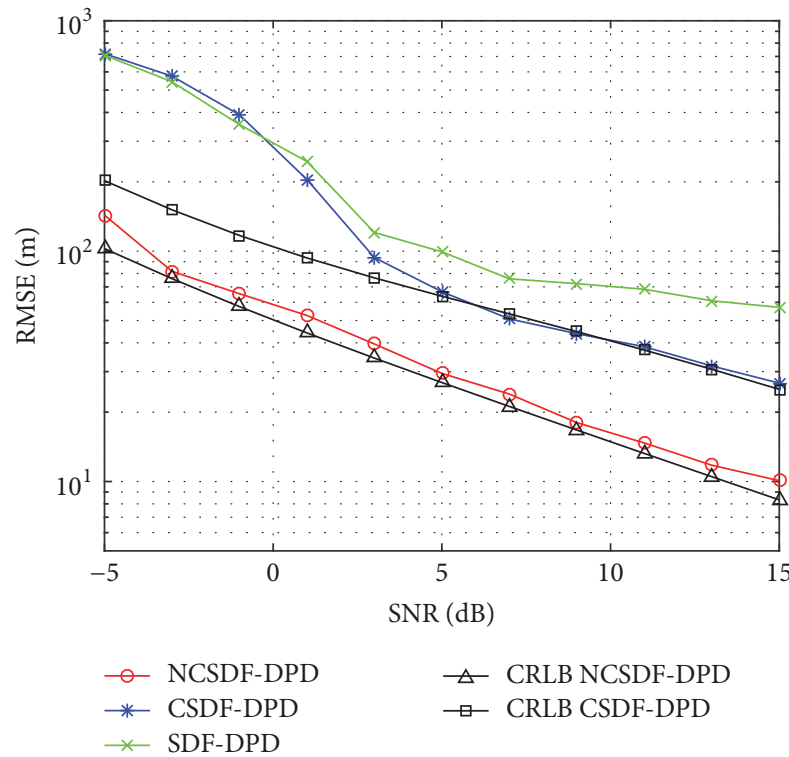

(a) Source 1

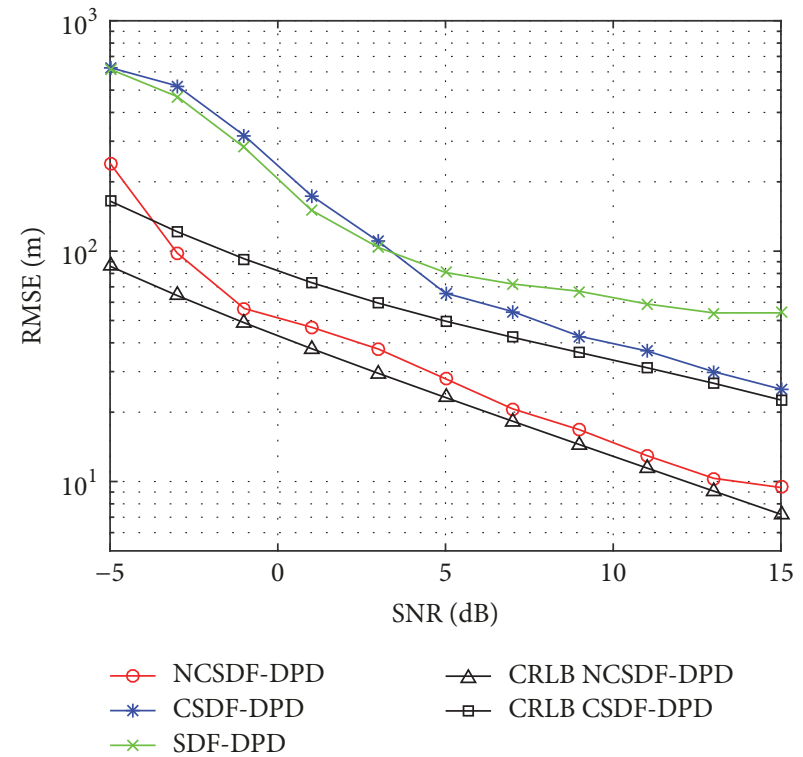

(b) Source 2

FIGURE 4: Square root of CRLBs and estimated RMSEs of three algorithms as a function of SNR: (a) source 1 and (b) source 2. 


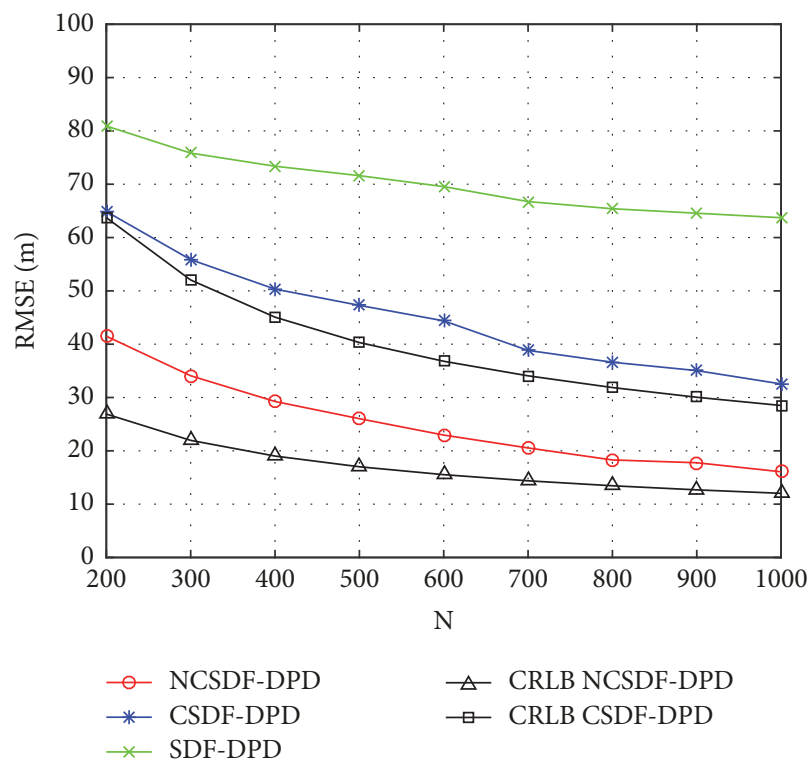

(a) Source 1

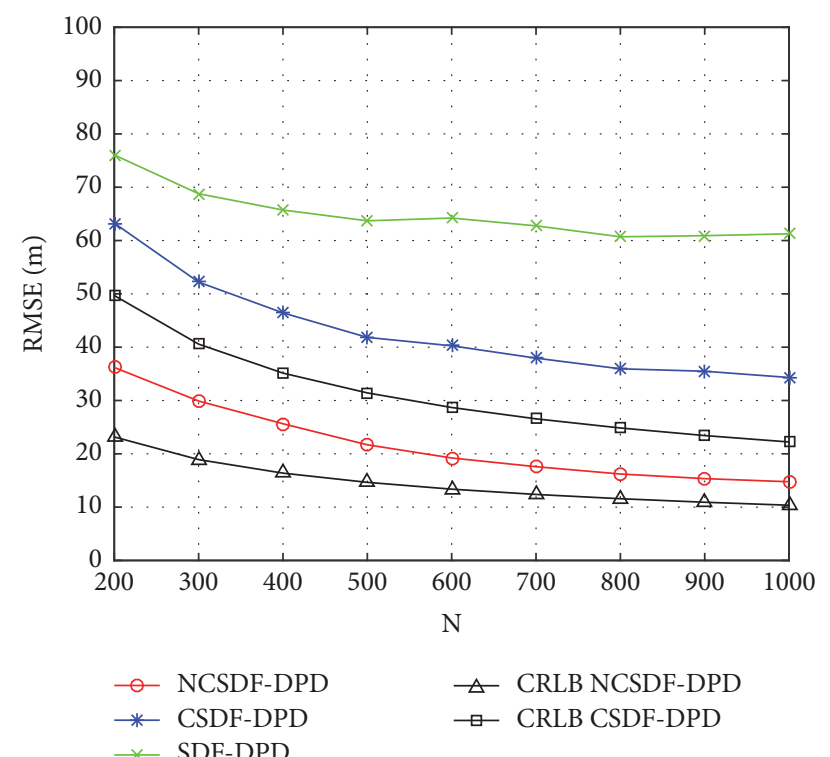

(b) Source 2

FIGURE 5: Estimated RMSEs of three algorithms as a function of $N$ : (a) source 1 and (b) source 2.

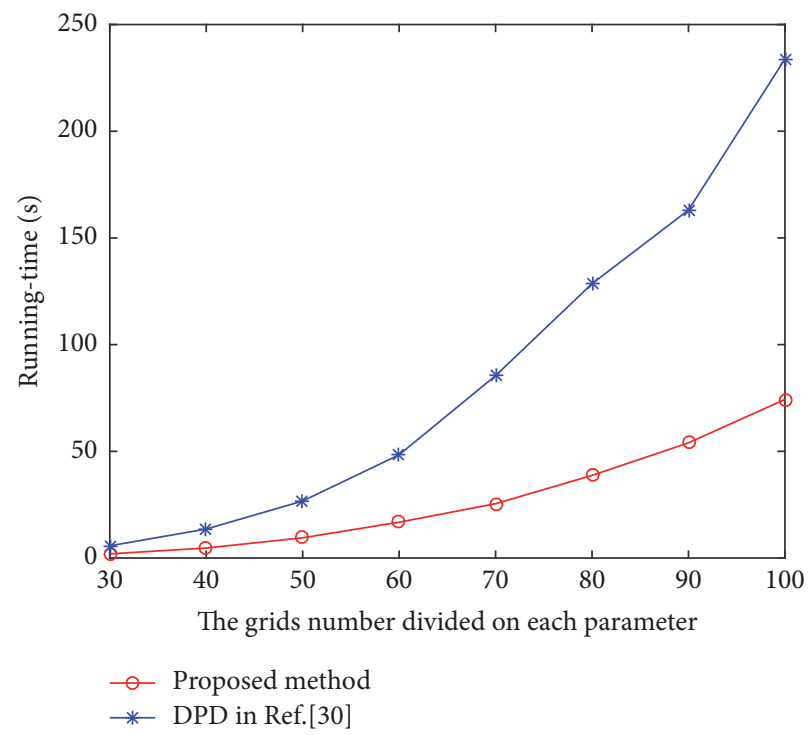

FIGURE 6: Running-times of different DPD methods.

dealing with multitarget localization under the condition of small samples, which is of considerable value in practical applications.

\section{Conflicts of Interest}

The authors declare no conflicts of interest.

\section{Acknowledgments}

The work was supported by the National Natural Science Foundation of China (Grant no. 61401513).

\section{References}

[1] Y. Shen and M. Z. Win, "Fundamental limits of wideband localization-part I: a general framework," IEEE Transactions on Information Theory, vol. 56, no. 10, pp. 4956-4980, 2010.

[2] Y. Han, Y. Shen, X.-P. Zhang, M. . Win, and H. Meng, "Performance limits and geometric properties of array localization," Institute of Electrical and Electronics Engineers Transactions on Information Theory, vol. 62, no. 2, pp. 1054-1075, 2016.

[3] Y. Wang, Y. Wu, and Y. Shen, "Multipath Effect Mitigation by Joint Spatiotemporal Separation in Large-Scale Array Localization," in Proceedings of the 2017 IEEE Global Communications Conference (GLOBECOM 2017), pp. 1-6, Singapore, December 2017. 
[4] Y. Feng and Z. Wanying, "Multiple model bernoulli particle filter formaneuvering target tracking," Journal of Electronics \& Information Technology, vol. 39, no. 3, pp. 634-639, 2017.

[5] Z. S. Yuan, H. T. Li, and X. H. Zhu, "A digital-analog hybrid random number generator based on memristor," Acta Physica Sinica, vol. 64, no. 24, Article ID 0240503, 2015.

[6] X. Xia and M. Liu, "Unified constrained cascade interactive multi-model filter and its application in tracking of manoeuvring target," Dianzi Yu Xinxi Xuebao/Journal of Electronics and Information Technology, vol. 39, no. 1, pp. 117-123, 2017.

[7] L. Xuedong, B. A. Bin, L. Zhiyu et al., "Sparse reconstruction time delay estimation algorithm based on backtracking filter," Acta Phys. Sin, vol. 65, no. 21, pp. 701-710, 2015.

[8] Y. U. Lee, "Distributed source modeling and direction-of-arrival estimation techniques," IEEE Transactions on Signal Processing, vol. 45, no. 4, pp. 960-969, 1997.

[9] B. T. Sieskul and S. Jitapunkul, "An asymptotic maximum likelihood for estimating the nominal angle of a spatially distributed source," AEÜ - International Journal of Electronics and Communications, vol. 60, no. 4, pp. 279-289, 2006.

[10] Z. Zheng, G. Li, and Y. Teng, "2D DOA estimator for multiple coherently distributed sources using modified propagator," Circuits, Systems and Signal Processing, vol. 31, no. 1, pp. 255270, 2012.

[11] T. Jantti, “The influence of extended sources on the theoretical performance of the MUSIC and ESPRIT methods: narrow-band sources," in Proceedings of the ICASSP-92: 1992 IEEE International Conference on Acoustics, Speech, and Signal Processing, pp. 429-432 vol.2, San Francisco, CA, USA, March 1992.

[12] T. Trump and B. Ottersten, "Estimation of nominal direction of arrival and angular spread using an array of sensors," Signal Processing, vol. 50, no. 1-2, pp. 57-69, 1996.

[13] Q. Wu, K. Wong, Y. Meng, and W. Read, "Doa Estimation of Point and Scattered Sources - Vet-Music," in Proceedings of the IEEE Seventh SP Workshop on Statistical Signal and Array Processing, pp. 365-368, Quebec, City, QC, Canada.

[14] S. Valaee, "Parametric Localization of Distributed Sources," IEEE Transactions on Signal Processing, vol. 43, no. 9, pp. 21442153, 1995.

[15] S. Shahbazpanahi, S. Valaee, and M. H. Bastani, "Distributed source localization using ESPRIT algorithm," IEEE Transactions on Signal Processing, vol. 49, no. 10, pp. 2169-2178, 2001.

[16] M. X. Yang, J. G. Li, and Z. Zheng, "Parameters estimation of coherently distributed non-circular signal based on sparse representation," Journal of Electronics and Information Technology, vol. 36, no. 1, pp. 164-168, 2014.

[17] L. Wan, G. Han, J. Jiang, J. J. P. C. Rodrigues, N. Feng, and T. Zhu, "DOA estimation for coherently distributed sources considering circular and noncircular signals in massive MIMO systems," IEEE Systems Journal, vol. 11, no. 1, pp. 41-49, 2017.

[18] X. Yang, G. Li, Z. Zheng, and L. Zhong, "2D DOA estimation of coherently distributed noncircular sources," Wireless Personal Communications, vol. 78, no. 2, pp. 1095-1102, 2014.

[19] J. Bosse, A. Ferreol, and P. Larzabal, "Performance analysis of passive localization strategies: Direct one step approach versus 2 steps approach," in Proceedings of the 2011 IEEE Statistical Signal Processing Workshop (SSP), pp. 701-704, Nice, France, June 2011.

[20] L. Zhi-Yu, R. Yan-Qing, B. Bin et al., "An improved direct position determination method based on correlation accumulation of short-time signals with variable velocity receivers," Acta Phys. Sin, vol. 66, no. 2, pp. 70-79, 2017.
[21] Z. Cheng, Y. Wang, and Y. Shen, "Direct position determination of multiple targets via reduced-dimension beamspace," in Proceedings of the 2017 IEEE International Conference on Communications Workshops, ICC Workshops 2017, pp. 10301035, fra, May 2017.

[22] D. Wang, R.-J. Zhang, and T. Zhang, "A Direct Position Determination Approach for Radio Emitters in Presence of Mutual Coupling," Tien Tzu Hsueh Pao/Acta Electronica Sinica, vol. 45, no. 5, pp. 1130-1138, 2017.

[23] A. J. Weiss, "Direct position determination of narrowband radio frequency transmitters," IEEE Signal Processing Letters, vol. 11, no. 5, pp. 513-516, 2004.

[24] A. J. Weiss and A. Amar, "Direct position determination of multiple radio signals," EURASIP Journal on Applied Signal Processing, vol. 2005, no. 1, pp. 37-49, 2005.

[25] B. Demissie, M. Oispuu, and E. Ruthotto, "Localization of multiple sources with a moving array using subspace data fusion," in Proceedings of the ISIF 11th International Conference on Information Fusion, p. 131, Cologne, Germany, May, 2008.

[26] L. Tzafri and A. J. Weiss, "High-resolution direct position determination using MVDR," IEEE Transactions on Wireless Communications, vol. 15, no. 9, pp. 6449-6461, 2016.

[27] D. Wang, Z. Gang, S. C. Yao et al., "A direct position determination algorithm for constant modulus signals with single moving observer," Acta Aeronautica et Astronautica Sinica, vol. 43, no. 5, pp. 1622-1633, 2016.

[28] O. Bar-Shalom and A. J. Weiss, "Efficient direct position determination of orthogonal frequency division multiplexing signals," IET Radar, Sonar \& Navigation, vol. 3, no. 2, pp. 101111, 2009.

[29] A. M. Reuven and A. J. Weiss, "Direct position determination of cyclostationary signals," Signal Processing, vol. 89, no. 12, pp. 2448-2464, 2009.

[30] O. Bar-Shalom and A. J. Weiss, "Direct emitter geolocation under local scattering," Signal Processing, vol. 117, article no. 5805, pp. 102-114, 2015. 


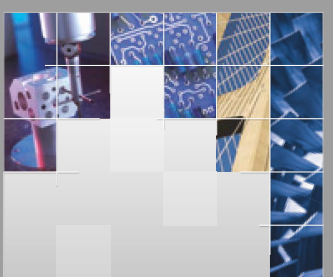

\section{Enfincering}
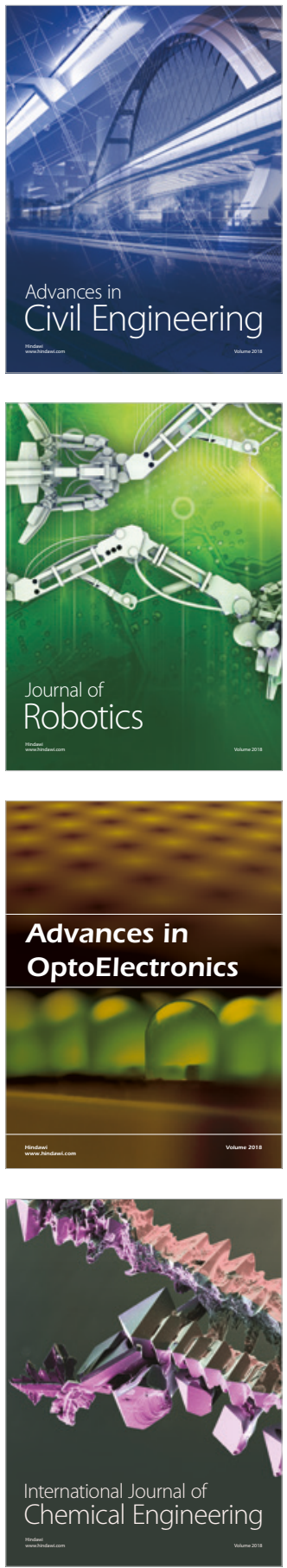

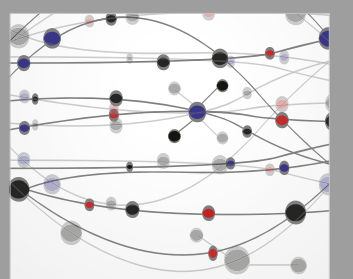

\section{Rotating \\ Machinery}

The Scientific World Journal

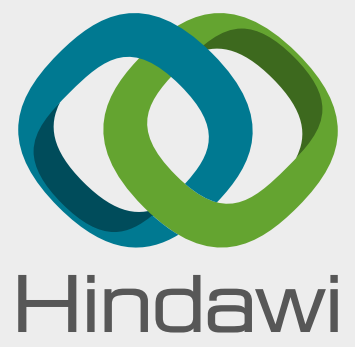

Submit your manuscripts at

www.hindawi.com
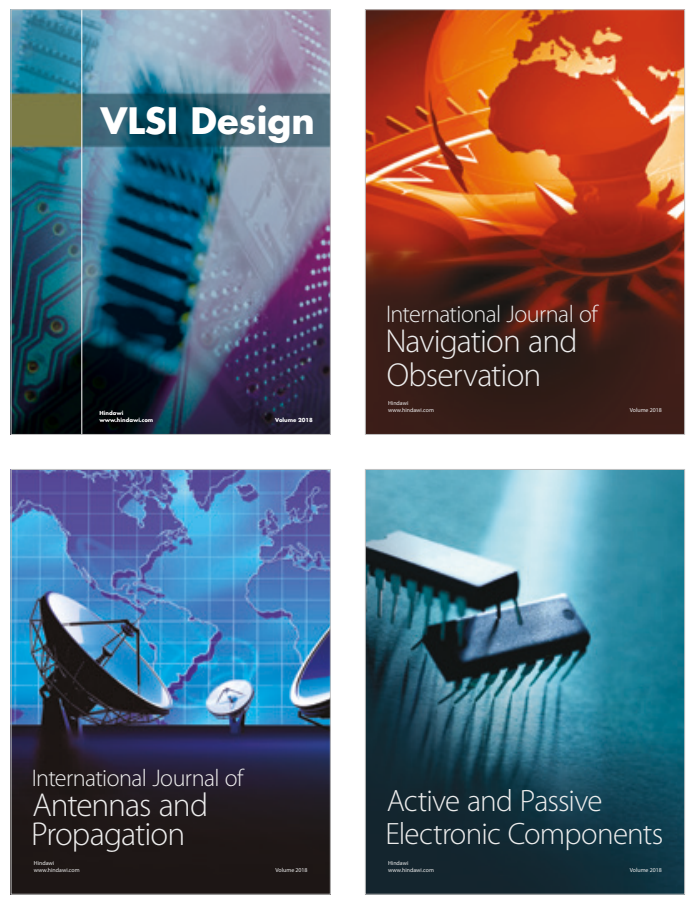
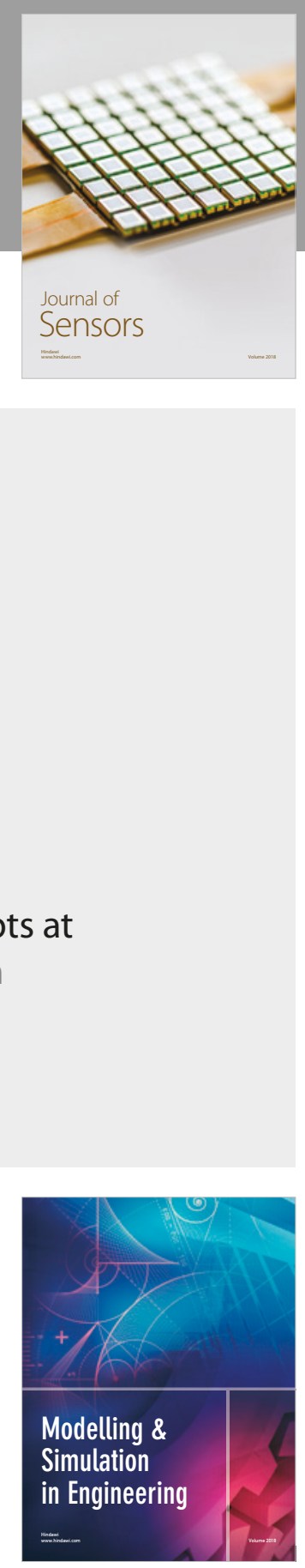

\section{Advances \\ Multimedia}
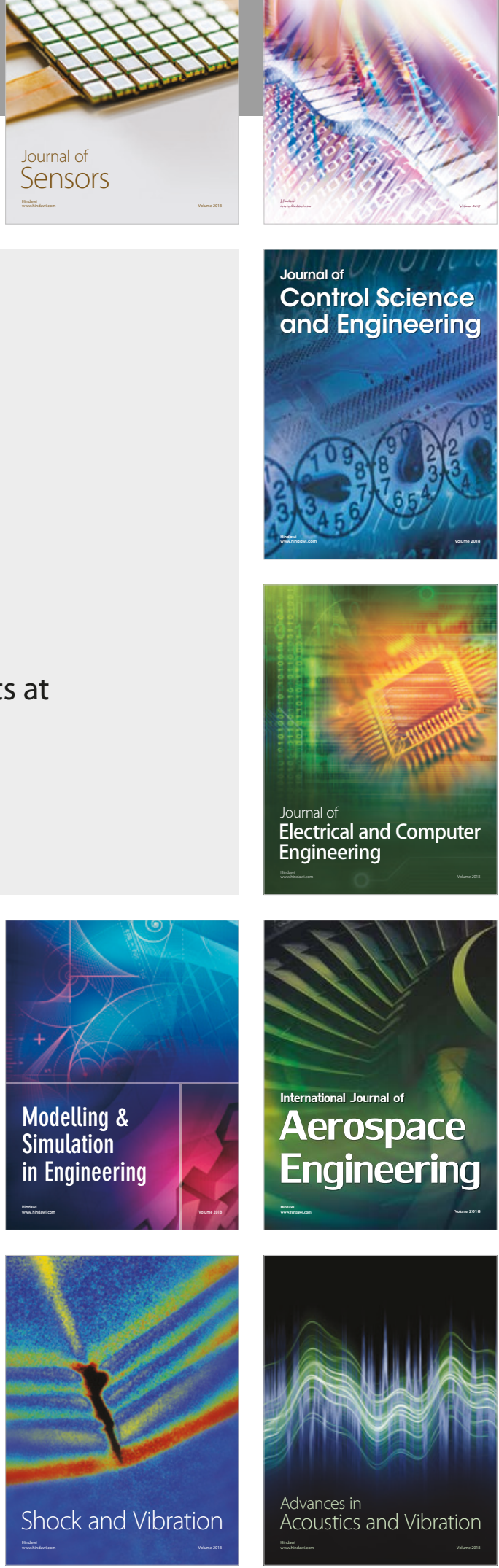